Artículo Original

\title{
Caracterización clínica y sociodemográfica de la población infantojuvenil entre 2 y 17 años con diagnóstico de deficiencia de extremidades superiores adquirida y/o congénita, en el Instituto Teletón Santiago
}

ISRAEL PATIÑO CABRERA ${ }^{1}$, MARÍA JOSÉ ESPINOZA², PAULA NAHUELHUAL ${ }^{4}$.

'Residente de Medicina

Física y Rehabilitación, Universidad del Desarrollo. 2Instituto Teletón Santiago,

Chile.

3Protex Integral

${ }^{4}$ Departamento de

Evaluación de Tecnologías

Sanitarias y Salud Basada en Evidencia. Ministerio de Salud, Chile.

Correspondencia a:

Dr. Israel Patiño

israel.patino@teleton.cl israelpatino85@hotmail.

com

Recibido: 11 de noviembre de 2020

Aceptado: 18 de junio de

\begin{abstract}
Clinical and sociodemographic characterization of the child and youth population between 2 and 17 years with a diagnosis of acquired or congenital upper limbs deficiency at Instituto Teletón Santiago
\end{abstract}

Introduction: Congenital and acquired deficiencies of the upper extremities are an important condition in the pediatric population, however, there is almost no information regarding the clinical and sociodemographic characteristics and those associated with the use of prostheses. Objective: Describe the clinical and sociodemographic characteristics of the child and adolescent population between 2 and 17 years old with a diagnosis of acquired and/or congenital upper limb deficiency, belonging to the Instituto Teletón Santiago (IT-S). Methods: A cross-sectional study was conducted in child and adolescent population between 2 and 17 years old, with a diagnosis of acquired and/ or congenital upper limb deficiency treated in the IT-S. A review of clinical records and a survey were carried out to obtain data on sociodemographic and clinical characteristics and characteristics associated with the use of prostheses. Results: 215 patients were included, $93.9 \%$ of congenital and $6.1 \%$ of acquired etiology. The most common level of deficiency was partial hand with $51.6 \%$, followed by transradial with $33.1 \%$. 33\% of the 215 patients included had a prescription for a prosthesis. $78.9 \%$ of the patients with a prosthesis prescription had a mechanical prosthesis and $18.3 \%$ had a 3D prosthesis. $53.5 \%$ used their prosthesis and $46.4 \%$ did not use it. $84.6 \%$ of the patients with $3 \mathrm{D}$ prostheses did not use them and an $83.3 \%$ of the patients with a partial hand level deficiency did not use their prosthesis. Conclusion: This study provides data of clinical importance, highlighting an early-onset prosthetic prescription associated with the clinical characteristics of the patients. Likewise, there is a high rate of non-use of 3D prostheses at the partial hand level.

Key words: limb deficiency, amputation, prosthetics, upper limb, children and adolescents. 


\section{RESUMEN}

Introducción: Las deficiencias congénitas y adquiridas de extremidades superiores son una condición importante en la población pediátrica, existe poca información respecto de sus características clínicas, sociodemográficas y las asociadas al uso de prótesis. Objetivo: Describir las características clínicas y sociodemográficas de la población infantojuvenil entre 2 y 17 años con diagnóstico de deficiencia de extremidades superiores adquirida y/o congénita, pertenecientes al Instituto Teletón Santiago (IT-S). Metodología: Estudio transversal, en población infantojuvenil entre 2 y 17 años, con diagnóstico de deficiencia de extremidades superiores, adquirida y congénita, que se atienden en el IT-S. Se realizó una revisión de fichas clínicas y encuesta para la obtención de datos de características sociodemográficas, clínicas y asociadas al uso de prótesis. Resultados: Se incluyeron 215 pacientes, 93,9\% de etiología congénita y $6,1 \%$ adquirida. El nivel de la deficiencia más frecuente fue parcial de mano con 51,6\%, seguido del transradial con 33,1\%. El 33\% de los 215 pacientes estudiados tuvieron prescripción de prótesis. De los pacientes con prescripción de prótesis, $78,9 \%$ correspondía a mecánica y $18,3 \%$ a prótesis 3D. El 53,5\% usaba su prótesis y el 46,4\% no la usaba. Respecto a las prótesis 3D, el 84,6\% no la usaban. En el nivel parcial de mano, el 83,3\% no usaban su prótesis. Conclusiones: Este estudio aporta datos de importancia clínica, destacando, una prescripción protésica de inicio temprano y asociada a las características clínicas de los pacientes. Así mismo, existe una alta tasa de no uso de las prótesis 3D, en el nivel parcial de mano.

Palabras clave: deficiencia de extremidades, amputación, prótesis, extremidad superior, niños y adolescentes.

\section{Introducción}

Las deficiencias de extremidades se caracterizan por la presencia de una o más alteraciones estructurales de las mismas ${ }^{6}$, se dividen en amputaciones adquiridas y deficiencias congénitas $^{3,7}$. En general, las estimaciones mundiales muestran 4-5 casos por 10.000 individuos/ año ${ }^{8}$. En Estados Unidos existen 6 casos por 10.000 nacidos vivos/año, con una relación de 2:1 para extremidades superiores respecto a inferiores ${ }^{2}$. En general, estos pacientes presentan limitaciones en sus actividades de la vida diaria y vocacionales ${ }^{9}$.

Respecto a datos locales, existen dos estudios muestrales realizados en el Instituto Teletón Santiago. En 2015, el estudio de Velasco y colaboradores reportó un $6,4 \%$ de amputaciones y/o malformaciones de extremidades de la población general atendida en Teletón Santiago ${ }^{10}$.
En 2019, el estudio de Flores y García, en una muestra de 57 pacientes mayores de 10 años con deficiencias congénitas de extremidades en general, se observó que las extremidades superiores estuvieron afectadas en un $68,5 \%$, siendo más frecuente la amputación transversal de antebrazo izquierdo ${ }^{11}$.

Las amputaciones adquiridas se refieren a la extirpación quirúrgica o espontánea, parcial o completa de una extremidad ${ }^{1}$, generalmente secundarias a trauma, infección, y pueden ser secundarias a neoplasia ${ }^{2,3}$. A su vez, las deficiencias congénitas se generan por una falla parcial o total en la formación de la extremidad durante el periodo de gestación ${ }^{3}$, como malformación, deformidad o displasia ${ }^{4}$. Malformación hace referencia a un defecto morfológico de un órgano o una región del cuerpo, debido a un proceso de desarrollo intrínsecamente anormal; a su vez, deformidad 
se refiere a una anormalidad en la forma o posición de una parte del cuerpo, secundaria a fuerzas mecánicas que alteran la morfogénesis; finalmente, displasia es una organización anormal de células en los tejidos, que resulta en una morfología anormal de una estructura ${ }^{5}$.

Para clasificar las deficiencias de extremidades, se usa el sistema de clasificación de la Sociedad Internacional de ortesis y la Sociedad Internacional de Prótesis y ortesis, propuesta en 1991. Esta clasificación define dos grupos, deficiencias transversales y longitudinales. Tanto en las deficiencias transversales como en las longitudinales, la afectación ósea se describe como total o parcial. En el caso de las deficiencias parciales, se puede describir como ausencia del tercio proximal, medial o distal del hueso afectado. En el caso de las deficiencias longitudinales, se describe el segmento óseo de proximal a distal, sabiendo que pueden existir elementos normales distalmente al segmento afectado. Las deficiencias transversales por su parte describen el segmento en el que termina la extremidad, sabiendo que no existe elementos óseos distales a este nivel ${ }^{12,13}$.

El manejo rehabilitador de las amputaciones adquiridas debe tener un inicio temprano. Cuando estas son electivas, se inicia con la evaluación y participación en la toma de decisión del nivel, posteriormente, el tratamiento debe enfocarse en el control del dolor, cuidado de la cicatriz, manejo protésico, entrenamiento de la funcionalidad, seguimiento y prevención de complicaciones ${ }^{14}$. En las deficiencias congénitas, las acciones se enfocan a optimizar el desarrollo psicomotor acorde para la edad, tratamiento quirúrgico y prescripción protésica en caso de requerirlo ${ }^{15,16}$.

Con respecto a la prescripción protésica, es necesario definir a una prótesis como un dispositivo externo que tiene como fin reemplazar total o parcialmente el segmento de una extremidad deficiente o ausente ${ }^{17}$. Existen prótesis cosméticas, mecánicas y mioeléctricas. Las mecánicas permiten funcionalidad (prensión, alcance, agarre, transporte y liberación) gracias a un dispositivo terminal de apertura o cierre voluntario. Las mioeléctricas utilizan señales electromiográficas de los músculos residuales para generar movimientos de los componentes $\operatorname{activos}^{18}$. Es importante la selección correcta de la prótesis y sus componentes, para alcanzar la función deseada. Se debe considerar la longitud residual de la extremidad, la condición de los tejidos y articulaciones, el control muscular remanente, los objetivos vocacionales, deseos cosméticos y el financiamiento ${ }^{14}$.

Existen problemas asociados a la aceptación y adherencia al uso de prótesis; algunas series han reportado un índice de rechazo entre $10 \%$ a $49 \%$. Las principales causas son: la falta de confort, problemas cutáneos, intolerancia por calor, el peso de la prótesis, la falta de aceptación cosmética y una pobre funcionalidad ${ }^{15,19}$. También se debe considerar la adherencia familiar y la integración social ${ }^{20}$. Por lo tanto, es importante tener un seguimiento con medidas de evaluación del desempeño y utilidad percibida por el paciente ${ }^{21,22}$.

En la revisión del sistema de gestión hospitalaria del IT-S, del 5 de diciembre de 2019, se pesquisaron 338 casos activos de deficiencias de extremidades superiores, congénitas y adquiridas $^{23}$. Sin embargo, no se conocen las características sociodemográficas, clínicas y las asociadas al uso de prótesis en estos pacientes.

Dado a lo anteriormente citado, se planteó como objetivo de este estudio, describir las características clínicas y sociodemográficas de la población infantojuvenil con diagnóstico de deficiencia de extremidades superiores, congénitas y/o adquiridas, en el Instituto Teletón Santiago.

\section{Materiales y Métodos}

\section{Tipo de estudio}

Estudio cuantitativo de tipo observacional con diseño retrospectivo descriptivo, desarrollado en el Instituto Teletón Santiago en el primer semestre de 2020 .

\section{Participantes}

La población de este estudio fueron 338 pacientes activos, que cumplían con los criterios de inclusión: diagnóstico de deficiencia de extremidades superiores, adquirida y congénita que se atienden en el IT-S, entre los 2 y 17 años, cuyos padres otorgaron el 
consentimiento informado de manera verbal durante una llamada telefónica. Los mismos que se obtuvieron de un sistema de información clínica institucional. Se excluyeron a los pacientes con diagnóstico no precisado, pacientes con diagnóstico de deficiencias de extremidades sin tipificar y pacientes con diagnósticos de deficiencias de extremidades superiores e inferiores concomitantemente.

\section{Variables}

Las variables se obtuvieron de la ficha clínica y de la encuesta aplicada. Las mismas que incluyeron: variables sociodemográficas (género, edad, escolarización, modalidad de escolarización y ubicación de la vivienda), de caracterización clínica (etiología, tipo, nivel, edad de presentación y número de extremidades afectadas) y variables relacionadas al uso de prótesis (prescripción, uso, razón de no uso, cantidad y frecuencia de uso, actividades de uso, edad de entrega, tipo de prótesis y reporte de nivel de satisfacción y utilidad).

\section{Instrumento de medición}

El instrumento fue una encuesta, elaborada por los autores y validada por 4 expertos en el tema, dos médicos fisiatras y dos terapeutas ocupacionales. Posteriormente, con sus recomendaciones, se elaboró una encuesta preliminar que fue pilotada con cuatro casos tomados al azar. Una vez aplicada la encuesta, se pidió a los familiares su opinión acerca de la comprensión y claridad de las preguntas. Con estos antecedentes, se determinó una encuesta definitiva, la misma que incluyó 11 preguntas, abiertas y cerradas, que permitieron obtener información de varios ámbitos como: características sociodemográficas, prescripción de prótesis, uso de la prótesis, días de uso, promedio de horas de uso, actividades en las que usa y la percepción de satisfacción y utilidad sobre el uso de prótesis.

El consentimiento informado se realizó de manera telefónica a los padres de los pacientes, se solicitó autorización para la aplicación de la encuesta y revisión de las fichas clínicas, información que posteriormente fue registrada en una base de datos. La encuesta se realizó por vía telefónica a los padres de los pacien- tes, con una duración promedio de 7 minutos. Tanto el cuestionario como la revisión de fichas se realizó entre el 7 de enero y el 9 de junio de 2020.

\section{Análisis estadístico}

Las variables fueron tabuladas en planilla Excel y procesadas en el software estadístico STATA 14, con medidas de frecuencia absoluta y relativa, valores absolutos y porcentajes, desviación estándar y promedio.

\section{Ética del estudio}

El proyecto fue aprobado por el Comité Ético Científico de la Sociedad Pro-Ayuda del Niño Lisiado en versión 2, con fecha 23/09/2019 (Certificado No 106/2019).

\section{Resultados}

\section{Características de los pacientes incluidos}

Las características de los pacientes incluidos en este estudio se detallan en la Figura 1. La tasa de contacto telefónico fue de $68,34 \%$, y los pacientes que aceptaron participar corresponden al 63,6\% del total de la población en estudio. Las características sociodemográficas de los pacientes incluidos se detallan en la Tabla 1. A su vez, las características clínicas de la población se encuentran detalladas en la Tabla 2.

\section{Características de la prótesis y su uso}

La gran mayoría de pacientes (67\%) no tuvo prescripción de prótesis, frente al 33\% que sí la tuvo. E1 78,9\% de prótesis prescritas fueron mecánicas, $18,3 \%$ prótesis $3 \mathrm{D}$, seguidas de prótesis cosmética y ortoprótesis con 1,4\% respectivamente. Del grupo de pacientes con prescripción de prótesis, el 53,5\% declara usarla frente al 46,4\% que no la usa. La gran mayoría de pacientes que, sí usan su prótesis, 60,5\% corresponde al nivel transradial, seguidas del $13,1 \%$ de los niveles desarticulado de codo y desarticulado de muñeca respectivamente, en menor proporciones menores los niveles parciales de mano, transhumeral y desarticulado de hombro. Por otro lado, respecto a los pacientes que no usan su prótesis, la gran mayoría corresponde a los niveles parcial de mano 


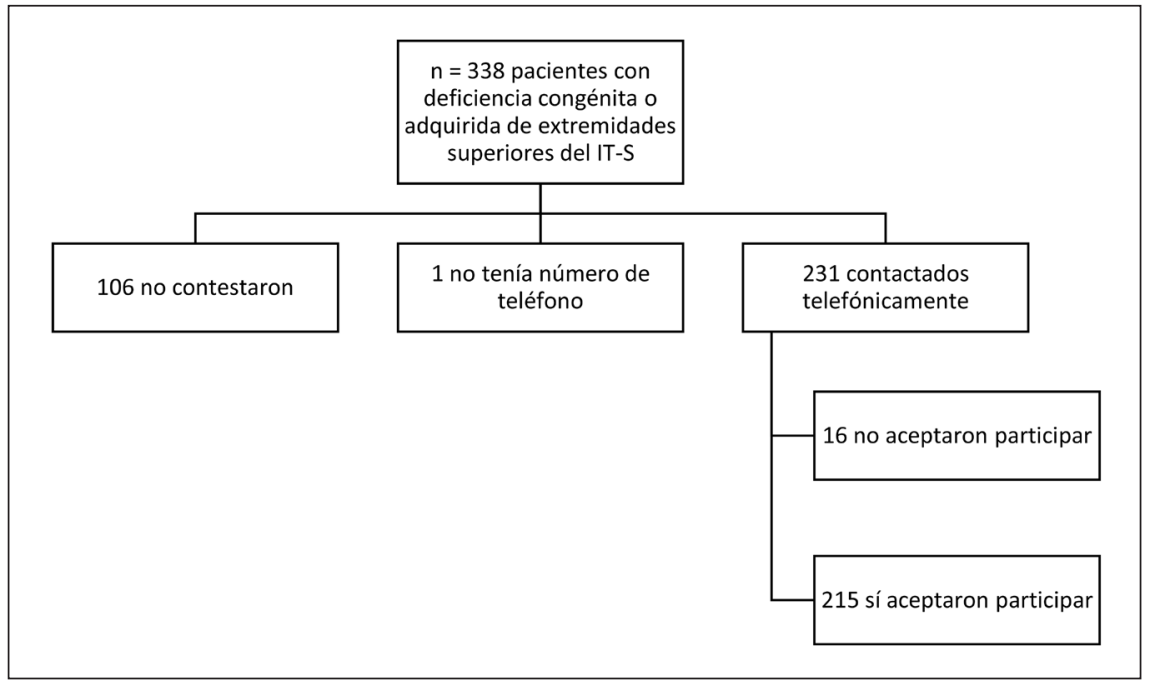

Figura 1. Características de la población incluida en el estudio.

Tabla 1. Características sociodemográficas de la población

\begin{tabular}{|lrc|}
\hline Característica & $\mathbf{n}$ & $\%$ \\
\hline Total & 215 & $100 \%$ \\
Género & & \\
$\quad$ Masculino & 111 & 51,6 \\
Femenino & 104 & 48,4 \\
Edad promedio edad en años (DE) & 8,5 & \\
Escolarización & & \\
Sí & 201 & 93,5 \\
No & 14 & 6,5 \\
Modalidad de escolarización & & \\
Colegio regular con PIE & 95 & 44,2 \\
Colegio regular sin PIE & 69 & 32,1 \\
Jardín infantil & 23 & 10,7 \\
Escuela especial / lenguaje & 7 & 3,2 \\
Prekinder / kinder & 4 & 1,9 \\
Sala cuna / guardería & 2 & 0,9 \\
Academia & 1 & 0,5 \\
Ubicación de la residencia & & \\
Urbana & 191 & 88,8 \\
Rural & 24 & 11,2 \\
\hline
\end{tabular}

DE: desviación estándar; PIE: proyecto de integración escolar

y transradial con un $30,3 \%$ respectivamente, seguidos del nivel desarticulado de muñeca con un $18,8 \%$ y en porcentajes menores los niveles desarticulados de codo, transhumeral y desarticulado de hombro (Tabla 5).
Tabla 2. Características clínicas de la población

\begin{tabular}{|lrr|}
\hline Característica & $\mathbf{n}$ & $\%$ \\
\hline Etiología & & \\
Congénita & 202 & 93,9 \\
Adquirida & 13 & 6,1 \\
Nivel de la condición & & \\
Parcial de mano & 111 & 51,6 \\
Transradial & 71 & 33,1 \\
Desarticulado de muñeca & 16 & 7,4 \\
Desarticulado de codo & 9 & 4,2 \\
Transhumeral & 6 & 2,8 \\
Desarticulado de hombro & 2 & 0,9 \\
Tipo de deficiencia & & \\
Longitudinal & 121 & 56,3 \\
Transversal & 94 & 43,7 \\
Extremidades afectadas & & \\
1 Extremidad & 189 & 87,9 \\
2 Extremidades & 25 & 11,6 \\
4 Extremidades & 1 & 0,5 \\
\hline
\end{tabular}

El tiempo promedio de horas de uso de la prótesis al día fue de 6,7 horas. Más del 50\% manifiesta usar la prótesis 7 días a la semana, sin diferencias en el tipo de actividades de uso. Respecto a las razones del no uso de prótesis, los detalles se encuentran especificados en la Tabla 3. Respecto a los niveles de satisfacción percibida por los padres sobre el uso de prótesis, la gran mayoría, el 23,6\% y el 63,2\% referían 
Tabla 3. Características de la prótesis y su uso

\begin{tabular}{|c|c|c|}
\hline Característica & $\mathbf{n}$ & $\%$ \\
\hline \multicolumn{3}{|l|}{ Prescripción de prótesis } \\
\hline No & 144 & 67 \\
\hline Sí & 71 & 33 \\
\hline \multicolumn{3}{|c|}{ Tipo de prótesis prescrita $(n=71)$} \\
\hline Mecánica & 56 & 78,9 \\
\hline $3 D$ & 13 & 18,3 \\
\hline Cosmética & 1 & 1,4 \\
\hline Ortoprótesis & 1 & 1,4 \\
\hline \multicolumn{3}{|l|}{ Uso de prótesis } \\
\hline Sí & 38 & 53,5 \\
\hline No & 33 & 46,4 \\
\hline \multicolumn{3}{|c|}{$\begin{array}{l}\text { Edad de entrega de la primera prótesis } \\
\text { en deficiencia congénita (años) }\end{array}$} \\
\hline Promedio & 2,1 & \\
\hline Mediana & 0,8 & \\
\hline Mínimo - máximo & 0,3 & 15,3 \\
\hline \multicolumn{3}{|c|}{ Número de días de uso de prótesis $(n=38)$} \\
\hline 7 días a la semana & 21 & 55,2 \\
\hline 5 días a la semana & 7 & 18,4 \\
\hline 3 días a la semana & 6 & 15,7 \\
\hline 2 días a la semana & 4 & 10,5 \\
\hline \multicolumn{3}{|l|}{ Horas de uso diarias } \\
\hline Promedio & 6,7 & \\
\hline Mínimo - máximo & 1 & 16 \\
\hline \multicolumn{3}{|c|}{$\begin{array}{l}\text { Actividades en las que usan la prótesis } \\
(n=38)\end{array}$} \\
\hline Escolar & 34 & 89,5 \\
\hline Deporte & 23 & 60,5 \\
\hline Juego & 22 & 57,9 \\
\hline AVD/autocuidado & 22 & 57,9 \\
\hline Terapia & 21 & 55,3 \\
\hline \multicolumn{3}{|c|}{ Razón no uso de prótesis $(n=33)$} \\
\hline No desea usar/no le gusta & 11 & 33,3 \\
\hline No tolera/le incomoda & 8 & 24,2 \\
\hline Limita su funcionalidad & 8 & 24,2 \\
\hline Es muy pesada & 4 & 12,2 \\
\hline En espera de entrega & 2 & 6,1 \\
\hline
\end{tabular}

niveles de "satisfecho" y "muy satisfecho", respectivamente. Así mismo, en cuanto a los niveles de utilidad de la prótesis percibidos por los padres, reportaron como "muy útil" un $55,2 \%$, "útil" un $18,4 \%$, "neutral" un $23,6 \%$ y "poco útil" un $2,6 \%$.
Tabla 4. Relación de tipo de prótesis y uso

\begin{tabular}{|lrrc|}
\hline $\begin{array}{l}\text { Tipo de } \\
\text { prótesis }\end{array}$ & Usa & $\begin{array}{c}\text { No } \\
\text { usa }\end{array}$ & $\begin{array}{c}\text { Tasa de Rechazo } \\
\text { por tipo de prótesis }\end{array}$ \\
\hline Mecánica & 35 & 21 & $37,5 \%$ \\
\hline Cosmética & 1 & 0 & $0 \%$ \\
\hline $3 D$ & 2 & 11 & $84,6 \%$ \\
\hline Ortoprótesis & 0 & 1 & $100 \%$ \\
\hline
\end{tabular}

En lo referente a la descripción entre el uso y el tipo de prótesis, observa que, del total de prótesis $3 \mathrm{D}$ prescritas ${ }^{13}$, el $84,6 \%$ no la usa; mientras que en las prótesis mecánicas el 62,5\% declara sí usar su prótesis y $37,5 \%$ no la usa (Tabla 4). Así mismo, con relación al nivel de deficiencia y la prescripción de prótesis, la mayoría de las deficiencias parciales de mano declaran no usar la prótesis, correspondiente al $83,3 \%$ de este nivel. Las características respecto al nivel de deficiencia y tipo de prótesis, uso de la prótesis y razón de no uso de prótesis, se detallan en la Tabla 5.

\section{Discusión}

En relación a los datos relevantes respecto a las características clínicas de los pacientes incluidos en este estudio vemos que, el origen de la deficiencia congénita fue considerablemente mayor respecto al adquirido, lo que concuerda con datos encontrados en la literatura para este grupo poblacional, así, en el estudio de Toda et al., se observa que, de la totalidad de pacientes estudiados, el $86,4 \%$ corresponde a deficiencias de origen congénito y solamente el $13,5 \%$ fueron adquiridas ${ }^{24}$. Por otro lado, respecto al nivel de presentación, vemos que en el estudio de Al-Worikat et al., el nivel más frecuente fue el transradial, seguido del nivel parcial de mano ${ }^{25}$.

Dentro de los hallazgos de nuestro estudio observamos un porcentaje de prescripción de prótesis del $33 \%$ y por el otro lado un $77 \%$ sin prescripción de prótesis. Al revisar la literatura, en el estudio de Kuyper et al., se observa que el porcentaje de prescripción de prótesis fue del $54,2 \%$, dentro de las cuales tuvieron mayor 
Tabla 5. Caracterización del nivel de deficiencia

\begin{tabular}{|lcccccc|}
\hline Nivel de deficiencia & $\begin{array}{c}\text { Parcial de } \\
\text { mano }\end{array}$ & Muñeca & $\begin{array}{c}\text { Trans- } \\
\text { radial }\end{array}$ & Codo & $\begin{array}{c}\text { Trans- } \\
\text { humeral }\end{array}$ & Hombro \\
Tipo de prótesis prescrita & 2 & 7 & 32 & 8 & 5 & 2 \\
$\quad$ Mecánica & 2 & 0 & 1 & 0 & 0 & 0 \\
$\quad$ Cosmética & 9 & 4 & 0 & 0 & 0 & 0 \\
3D & 1 & 0 & 0 & 0 & 0 & 0 \\
$\quad$ Ortoprótesis & & & & & & \\
Uso de prótesis y características clínicas & 2 & 5 & 23 & 5 & 2 & 1 \\
Sí & 10 & 6 & 10 & 3 & 3 & 1 \\
No & & & & & & \\
Razón de no uso & 1 & 1 & 4 & 1 & 1 & 0 \\
No tolera & 6 & 0 & 2 & 0 & 0 & 0 \\
Limita función & 0 & 5 & 4 & 1 & 1 & 0 \\
No desea usar & 1 & 0 & 0 & 1 & 1 & 1 \\
Es muy pesada & 2 & 0 & 0 & 0 & 0 & 0 \\
$\quad$ En espera de entrega & & & & & & \\
\hline
\end{tabular}

prescripción los niños con nivel transradial y los que no tuvieron prescripción fueron los de etiología congénita longitudinal ${ }^{26}$. Esta diferencia está dada por las características clínicas de la deficiencia.

En nuestros hallazgos las prótesis mayormente prescritas fueron las mecánicas. En el estudio de Kuyper et al., las prótesis más prescritas fueron las cosméticas, seguidas de las mecánicas ${ }^{26}$, lo que nuevamente nos lleva a suponer que esta diferencia está dada por las características clínicas de la población en estudio. Así mismo, el nivel que mayor prescripción de prótesis mecánicas recibió en nuestro estudio fue el transradial, a diferencia de las prótesis 3D que fueron prescritas en los niveles distales, parciales de mano y desarticulado de muñeca. En la literatura se describe que la indicación protésica debe ser de carácter individual y obedecerá a las condiciones clínicas y sociales de los pacientes ${ }^{27}$.

En nuestros hallazgos, respecto a la edad promedio de entrega de la primera prótesis, vemos una mediana de 0,8 años. La revisión sistemática de Meurs, incluyó 4 estudios de cohorte retrospectivo, donde observaron que la edad de entrega de la primera prótesis en pacientes con deficiencias congénitas de extremidades superiores fue antes de los dos años de $\operatorname{edad}^{28}$. Por otro lado, Kuyper et al., determinaron que la media de entrega de la primera prótesis fue a los 2,6 años ${ }^{26}$. En general, la literatura recomienda que el inicio del manejo protésico sea a los 6 meses de edad, cuando empieza el sedente y tiene como función lograr el apoyo y equilibrio ${ }^{27}$. Algunos estudios consideran que un manejo protésico temprano conducirá a una mejor adherencia y resultados funcionales ${ }^{27,29}$. Por lo tanto, en este estudio observamos que la edad de entrega de la primera prótesis se apega a lo encontrado en la literatura.

En referencia al uso de prótesis, el porcentaje de uso fue de $53,5 \%$ y el de no uso fue de $46,4 \%$. Porcentajes que se correlacionan con un estudio de Dabaghi, que encontró una tasa de rechazo de un $49 \%{ }^{15}$. Así mismo, en un estudio de Wagner, con 489 pacientes entre los 2 a los 20 años, se reportó una tasa de rechazo del $34 \%{ }^{19}$. A su vez, respecto a las razones de no uso de la prótesis, encontramos las siguientes: que no desea usarla, disconfort o no tolerancia, que limita la funcionalidad y que es muy pesada. Estos datos se correlacionan con lo reportado por Wagner, que identificó como causas de no uso: prótesis no funcional, no confortable, que no les gustaba y que tenía mal ajuste ${ }^{19}$. Por lo tanto, vemos que los datos de este estudio son muy similares a los reportados en la literatura. Con relación 
al tipo de prótesis y su uso, se detectó que las prótesis 3D no son usadas por los pacientes en un porcentaje de $84,6 \%$. En el estudio de Dote y colaboradores, acerca del efecto de la prótesis 3D en la funcionalidad de adolescentes con deficiencia congénita parcial de mano, se reportó que este tipo de prótesis no mejora la funcionalidad ${ }^{30}$. Datos que podrían explicar el alto porcentaje de rechazo de las prótesis $3 \mathrm{D}$ reportada en nuestro estudio.

Respecto a la frecuencia de uso de la prótesis, lo hallado en este estudio, indica que más del $50 \%$ de pacientes que usaban su prótesis, lo hacían todos los días, con un promedio de 6,7 horas al día. En el estudio de Alvial, acerca de la prevalencia de inserción laboral en pacientes amputados mayores de 18 años, se observó que el $87,1 \%$ de pacientes que usaban prótesis, lo hacían por más de 7 horas al día, todos los días ${ }^{31}$, siendo esta población laboralmente activa, a diferencia de nuestra población que son menores de edad y estudiantes en su gran mayoría, sin embargo, nos permite tener un parámetro de los comportamientos respecto al promedio de horas de uso de la prótesis en diferentes contextos funcionales y de edades. Por otro lado, Egermann, en su estudio con pacientes usuarios de prótesis mioeléctricas, consideró como uso exitoso de la prótesis a un tiempo mayor a dos horas al día ${ }^{32}$. En el estudio de Crandall y Tomhave, se reportó un tiempo promedio de uso de prótesis de extremidades superiores, de 9,72 horas por día ${ }^{33}$. De acuerdo con lo mencionado, podemos ver que existe variabilidad respecto al tiempo de uso en distintas poblaciones, lo que puede ser debido a las diferencias en las edades, características clínicas y necesidades funcionales en el uso de prótesis. Sin embargo, en nuestro estudio observamos que el tiempo de uso está dentro de los parámetros habituales y podríamos considerarlo como un tiempo exitoso. En relación con las actividades en las que se usaba la prótesis, la actividad con mayor porcentaje de uso fue durante la actividad escolar.

Respecto al uso de prótesis y el nivel de deficiencia parcial de mano, se reportó no uso de la prótesis en un 83,3\%. Así mismo, en este nivel, de las 12 prótesis prescritas,
9 de ellas fueron 3D. Lo que corrobora aún más lo mencionado en el estudio de Dote, que en este nivel las prótesis $3 \mathrm{D}$ no mejoran la funcionalidad ${ }^{30}$ y explicaría su rechazo. Estos datos, de igual manera se reafirman con los resultados del estudio de Postema, que reporta que los dos tercios de los niños con amputación debajo de la muñeca rechazaron la prótesis ${ }^{34}$. Con respecto a los niveles de satisfacción y utilidad en relación con el uso de prótesis, se encontraron porcentajes altos en niveles de muy satisfecho y útil en las respuestas referidas por los padres.

Dentro de las limitaciones de este proyecto, se consideran que, el proyecto se desarrolló en medio de la pandemia por SARS-CoV-19, con lo que existió algún grado de reticencia a brindar información por parte de los familiares durante las llamadas telefónicas. Así mismo, existió un porcentaje de rechazo a participar del proyecto del $7 \%$ del total de familiares contactados. Por otro lado, 107 pacientes no fueron contactados, lo que representó el 31,6\% de la población total en estudio. Limitaciones del tipo de estudio.

Respecto a las proyecciones de este estudio, se considera que esta investigación sienta las bases para estudios analíticos respecto a esta población.

\section{Conclusiones}

Este estudio reveló datos de importancia clínica. Destacando, que la prescripción protésica es de inicio temprano y según las características clínicas del paciente. Por otro lado, vemos una alta tasa de rechazo en el nivel parcial de mano y en las prótesis 3D. Datos que servirán de base para mejorar las opciones terapéuticas en este grupo.

\section{Agradecimientos}

Los autores agradecen a la Subdirección de Investigación de Teletón Chile y al cuerpo docente de la Universidad del Desarrollo, por su contribución y apoyo durante el proceso de este proyecto. 


\section{Conflicto de interés}

Los autores declaran no tener conflictos de interés tales como haber trabajado, asesorado, tener participaciones, haber recibido honorarios, haber actuado como testigo pericial pagado, haber solicitado/registrado patentes o haber recibido subvenciones u otros fondos, relacionados con lo presentado en este estudio.

\section{Referencias}

1. Kohler F, Cieza A, Stucki G, Geertzen J, Burgers H, Dillon $\mathrm{M}$, et al. Developing Core Sets for persons following amputation based on the International Classification of Functioning, Disability and Health as a way to specify functioning. Prosthet Orthot Int. 2009; 33 (2): 117-29.

2. Le J, Scott P. Pediatric Limb Differences and Amputations. Phys Med Rehabil Clin N Am. 2015; 26: 95-108.

3. Modrcin A, McLaughlin M, Luetke M. Pediatric Limb Deficiencies. In: Alexander M, Matthews D, editors. Pediatric Rehabilitation Principles and Practice. Demos Medi. New York; 2015. p. 307-35.

4. Tonkin MA, Oberg KC. The OMT Classification of Congenital Anomalies of the Hand and Upper Limb. Hand Surg. 2015; 20 (3): 336-42.

5. Syvanwn J. Congenital Lower Limb Deficiencies in Finland. Risk Factor, prevalence, associated anomalies and treatment. Ann Univ Turku MEDICA - Odontol. 2020; 1476.

6. Araujo E, Buchalla C. The use of the International Classification of Functioning, Disability and Health in health surveys: a reflexion on its limits and possibilities. Rev bras epidemiol. 2015; 18 (3): 720-4.

7. Pereira J, Pereira L. Epidemiological Data of Amputations in Children. Clin Pediatr Dermatology. 2016; 2 (2): 1-3.

8. Zúñiga J, Carson A, Peck J, Kalina T, Krivastava R, Peck K. The development of a low-cost three-dimensional printed shoulder, arm, and hand prostheses for children. Prosthetics Orthot Int. 2016; 1-5.

9. Dillingham T, Braza D. Upper Limb Amputations. In: Frontera W, Silver J, Rizzo T, editors. Essentials of Physical Medicine and Rehabilitation Musculoskeletal Disorders, Pain, and Rehabilitation. Third Edit. Philadelphia; 2015. p. 596-602.

10. Velasco A, García D, San Martín P, Solís F. No Dolor fantasma en niños y jóvenes amputados adquiridos: pre- valencia y características clínicas Title. Rehabil Integr. 2015; 10 (1): 8-16.

11. Flores E, García D. Dolor fantasma en niños y jóvenes amputados congénitos: prevalencia y características clínicas. Rehabil Integr. 2019; 14 (1): 22-9.

12. Day HJB. The ISO/ISPO classification of congenital limb deficiency. Prosthet Orthot Int. 1991; 15: 67-9.

13. Harder J, Krajbich J. The Child With a Limb Deficiency. In: Atlas of Amputations and Limb Deficiencies Surgical, Prosthetic, and Rehabilitation Principles. Fourth Edi. 2016. p. 751-8.

14. Esquenazi A. Upper Limb Amputation, Rehabilitation, \& Prosthetic Restoration. In: McGraw-Hill, editor. CURRENT Diagnosis \& Treatment: Physical Medicine \& Rehabilitation. First Edit. Philadelphia; 2015. p. 453-9.

15. Dabaghi-Richerand A, Haces-García F, Capdevila-Leonori R. Prognostic factors of a satisfactory functional result in patients with unilateral amputations of the upper limb above the wrist that use an upper limb prosthesis. Rev Esp Cir Ortop Traumatol. 2014; 11 (001): 18884415.

16. Scott B. Physical Medicine and Rehabilitation Management of Children with Congenital Anomalies of the Upper Extremity. In: Springer, editor. Congenital Anomalies of the Upper Extremity Etiology and Management. First Edit. New York; 2015. p. 51-7.

17. World Health Organization. Geneva: World Health Organization standards for prosthetics and orthotics. Vol. Licence: C. 2017. 1-84 p.

18. Zheng J, Kalpakjian C, Martínez M, Chestek C, Deanna G. Priorities for the design and control of upper limb prostheses: A focus group study Title. Disabil Health J. 2019;:S1936-6574 (19): 30054-8.

19. Wagner L, Bagley A, James M. Reasons for prosthetic rejection by children with unilateral congenital transverse forearm total deficiency. J Prosthetics Orthot. 2007; 19 (2): 51-4.

20. Yazdani M, Kamali M. Evaluation of important factors in accepts or reject of upper limb prosthesis in children. Glob Journals Adv Pure Appl Sci. 2015; 7: 65-72.

21. Linder H, Natterlund B, Hermansson L. Upper limb prosthetic outcome measures: Review and content comparison based on International Classification of Functioning, Disability and Health. Prosthet Orthot Int. 2010; 43 (2): 109-28.

22. Lindner H, Hiyoshi A, Hermansson L. Relation between capacity and performance in paediatric upper limb prosthesis users. Prosthet Orthot Int. 2017; 42 (1): 1420. 
23. Teletón Santiago. Cub OLAP Deficiencias de Extremidades Superiores. 2019.

24. Toda M, Chin T, Shibata Y, Mizobe F. Use of Powered Prosthesis for Children with Upper Limb Deficiency at Hyogo Rehabilitation Center. PLoS One. 2015; 10 (6): e0131746.

25. Al-Worikat A, Dameh W. Children with limb deficiencies: Demographic characteristics. Prosthet Orthot Int. 2008; 32 (1): 23-8.

26. Kuyper M, Breedijk M, Mulders A, Post M, Prevo A. Prosthetic management of children in the Netherlands with upper limb deficiencies. Prosthet Orthot Int. 2001; 25 (3): 228-34

27. Kanas J. Prosthetic Management for Pediatric Upper Limb Deficiency. Springer Sci Media. 2014; 85: 1-25.

28. Meurs M, Maathuis C, Lucas C, Hadders-Algra M, Meurs M, Maathuis CGB, et al. Prescription of the first prosthesis and later use in children with congenital unilateral upper limb deficiency: A systematic review. Prosthet Orthot Int. 2006; 30 (2):165-73.

29. Krebs D, Edelstein J, Thornby M. Prosthetic management of children with limb deficiencies. Phys Ther.
1991; 71 (12): 920-34.

30. Dote J, Nahuelhual P, Cubillos R, Fuentes G, Zúñiga J. Funcionalidad de prótesis de mano impresa en 3D en adolescentes con amputación congénita parcial de mano: una serie de casos. Rev Chil Pediatr. 2020; 91 (3): 410-6.

31. Alvial P, Espinoza MJ, Moyano A, Solís F, San Martín P. Prevalencia de inserción laboral en la adultez de pacientes amputados antes de los 18 años de edad y factores clínico-demográficos asociados. Instituto Teletón Santiago 2012-2013. Rehabil Integr. 2014; 9 (1): 35-43.

32. Egermann M, Kasten P, Thomsen M. Myoelectric hand prostheses in very young children. Int Orthop. 2009; 33 (4): 1101-5.

33. Crandall R, Tomhave W. Pediatric Unilateral BelowElbow Amputees: Retrospective Analysis of 34 Patients Given Multiple Prosthetic Options. J Pediatr Orthop. 2002; 22: 380-3.

34. Postema K, Van der Donk V, Van Limbeek J, Rijken R, Poelma M. Prosthesis rejection in children with a unilateral congenital arm defect. Clin Rehabil. 1999; 13: 243-9. 